\title{
ON THE NUMERICAL EVALUATION OF LEGENDRE'S CHI-FUNCTION
}

\author{
J. BOERSMA AND J. P. DEMPSEY
}

\begin{abstract}
Legendre's chi-function, $\chi_{n}(z)=\sum_{k=0}^{\infty} z^{2 k+1} /(2 k+1)^{n}$, is reexpanded in a power series in powers of $\log z$. The expansion obtained is well suited for the computation of $\chi_{n}(z)$ in the two cases of real $z$ close to 1, and $z=e^{i \alpha}, \alpha \in \mathbb{R}$. For $n=2$ and $n=3$, the present computational procedure is shown to be superior to the procedure recently proposed by Dempsey, Liu, and Dempsey, which uses Plana's summation formula.
\end{abstract}

\section{INTRODUCTION}

Recently, Dempsey, Liu, and Dempsey [2] presented a procedure for the numerical evaluation of the series

$$
\begin{array}{cl}
\sum_{k=0}^{\infty} \frac{A^{2 k+1}}{(2 k+1)^{2}}, & \sum_{k=0}^{\infty} \frac{A^{2 k+1}}{(2 k+1)^{3}} \quad(0<A \leq 1), \\
\sum_{k=0}^{\infty} \frac{\sin (2 k+1) \alpha}{(2 k+1)^{2}}, & \sum_{k=0}^{\infty} \frac{\cos (2 k+1) \alpha}{(2 k+1)^{3}} \quad(0 \leq \alpha \leq \pi),
\end{array}
$$

which arise in the mathematical analysis of unilateral plate contact problems [6, Appendix B]. These series are slowly convergent, particularly if $A$ is close to 1 . To improve the convergence, the procedure in [2] uses Plana's summation formula, which in turn requires the computation of several definite integrals by Romberg integration.

In this paper we develop a computational procedure which appears to be considerably simpler. The series (1.1) are treated as special cases $(n=2$ and $n=3$ ) of Legendre's chi-function of order $n$, defined by

$$
\chi_{n}(z)=\sum_{k=0}^{\infty} \frac{z^{2 k+1}}{(2 k+1)^{n}}, \quad|z| \leq 1, n=2,3,4, \ldots
$$

Here the terminology and notation have been adopted from Lewin [5, p. 283]. The chi-function can also be expressed as

$$
\chi_{n}(z)=\frac{1}{2}\left[\mathrm{Li}_{n}(z)-\mathrm{Li}_{n}(-z)\right]=\mathrm{Li}_{n}(z)-2^{-n} \mathrm{Li}_{n}\left(z^{2}\right),
$$

where $\mathrm{Li}_{n}$ stands for the polylogarithm of order $n$, defined by [5, p. 282]

$$
\operatorname{Li}_{n}(z)=\sum_{k=1}^{\infty} \frac{z^{k}}{k^{n}}, \quad|z| \leq 1, n=2,3,4, \ldots
$$

Received by the editor June 26, 1991 and, in revised form, August 29, 1991. 1991 Mathematics Subject Classification. Primary 65B10; Secondary 33E20. 
On setting $z=e^{i \alpha}, \alpha \in \mathbb{R}$, in (1.3), we introduce the notations

$$
C_{n}(\alpha)=\sum_{k=0}^{\infty} \frac{\cos (2 k+1) \alpha}{(2 k+1)^{n}}, \quad S_{n}(\alpha)=\sum_{k=0}^{\infty} \frac{\sin (2 k+1) \alpha}{(2 k+1)^{n}}
$$

for the real and imaginary parts of $\chi_{n}\left(e^{i \alpha}\right)$. Obviously, the series (1.2) are identical to $S_{2}(\alpha)$ and $C_{3}(\alpha)$. It is shown below that the Fourier series (1.6) for $C_{2 n}(\alpha)$ and $S_{2 n+1}(\alpha)$ are summable in closed form, the sum being a polynomial in $\alpha$. The functions $S_{2 n}(\alpha)$ and $C_{2 n+1}(\alpha)$ can be expressed as

$$
\begin{aligned}
& S_{2 n}(\alpha)=\mathrm{Cl}_{2 n}(\alpha)-2^{-2 n} \mathrm{Cl}_{2 n}(2 \alpha), \\
& C_{2 n+1}(\alpha)=\mathrm{Cl}_{2 n+1}(\alpha)-2^{-2 n-1} \mathrm{Cl}_{2 n+1}(2 \alpha),
\end{aligned}
$$

where $\mathrm{Cl}_{n}$ denotes the generalized Clausen function, given by [5, p. 281]

$$
\mathrm{Cl}_{2 n}(\theta)=\sum_{k=1}^{\infty} \frac{\sin (k \theta)}{k^{2 n}}, \quad \mathrm{Cl}_{2 n+1}(\theta)=\sum_{k=1}^{\infty} \frac{\cos (k \theta)}{k^{2 n+1}} .
$$

In $\S 2$ of this paper, Legendre's chi-function (1.3) is reexpanded in a power series in powers of $\log z$. The expansion obtained and the corresponding expansions for $C_{n}(\alpha)$ and $S_{n}(\alpha)$ are rapidly convergent for real $z$ close to 1 and for $0 \leq \alpha \leq \pi / 2$, and are eminently suitable for the computation of $\chi_{n}(z)$, $C_{n}(\alpha)$, and $S_{n}(\alpha)$. In $\S 3$ the expansions are specialized for the cases $n=2$ and $n=3$, and are then used in the numerical evaluation of the series (1.1) and (1.2). The present approach is shown to be more effective than the procedure in [2] based on Plana's summation formula. Yet another computational procedure has been developed by Gautschi [4]. In his approach the function $\chi_{n}(z)$ (or $R_{n}(z)$ in the notation of [4]) is expressed as a Stieltjes transform which is then evaluated by backward recursion. As for the numerical evaluation of the series (1.1), our procedure and Gautschi's are comparable in efficiency, except near (and below) $A=e^{-\pi}$ and in the vicinity of $A=1$, where Gautschi's, resp. our, procedure is clearly superior. For the series (1.2) our procedure is believed to be more efficient than Gautschi's, since the latter involves complex arithmetic.

\section{EXPANSIONS FOR $\chi_{n}(z), C_{n}(\alpha)$, AND $S_{n}(\alpha)$}

In terms of Lerch's transcendent $\Phi(z, s, v)[3, \S 1.11]$, defined by

$$
\Phi(z, s, v)=\sum_{k=0}^{\infty} \frac{z^{k}}{(v+k)^{s}}, \quad|z|<1, v \neq 0,-1,-2, \ldots,
$$

the function $\chi_{n}(z)$ can be expressed as

$$
\chi_{n}(z)=2^{-n} z \Phi\left(z^{2}, n, \frac{1}{2}\right)
$$

We now employ the series expansion from [3, equation 1.11(9)] to obtain

$$
\begin{aligned}
\chi_{n}(z)= & 2^{-n} \sum_{\substack{k=0 \\
k \neq n-1}}^{\infty} \zeta\left(n-k, \frac{1}{2}\right) \frac{\left(\log z^{2}\right)^{k}}{k !} \\
& +\frac{\left(\log z^{2}\right)^{n-1}}{2^{n}(n-1) !}\left[\psi(n)-\psi\left(\frac{1}{2}\right)-\log \left(\log \left(\frac{1}{z^{2}}\right)\right)\right],
\end{aligned}
$$

$\left|\log z^{2}\right|<2 \pi$. 
Here, $\psi(s)$ denotes the logarithmic derivative of the gamma function [3, §1.7], i.e., $\psi(s)=\Gamma^{\prime}(s) / \Gamma(s)$. By setting $m=n-k$, the generalized zeta function $\zeta\left(m, \frac{1}{2}\right)$ is evaluated by means of [3, equations $\left.1.10(1),(11), 1.13(14),(15)\right]$ :

$$
\begin{aligned}
& \zeta\left(m, \frac{1}{2}\right)=\sum_{k=0}^{\infty} \frac{1}{\left(k+\frac{1}{2}\right)^{m}}=\left(2^{m}-1\right) \zeta(m), \quad m=2,3,4, \ldots, \\
& \zeta\left(-2 m, \frac{1}{2}\right)=-\frac{B_{2 m+1}\left(\frac{1}{2}\right)}{2 m+1}=0, \quad m=0,1,2, \ldots, \\
& \zeta\left(-2 m-1, \frac{1}{2}\right)=-\frac{B_{2 m+2}\left(\frac{1}{2}\right)}{2 m+2}=-2(-1)^{m}(2 m+1) ! \sum_{r=1}^{\infty} \frac{(-1)^{r}}{(2 \pi r)^{2 m+2}} \\
&=\frac{2(-1)^{m}(2 m+1) !}{(2 \pi)^{2 m+2}}\left(1-2^{-2 m-1}\right) \zeta(2 m+2), \\
& \quad m=0,1,2, \ldots,
\end{aligned}
$$

where $\zeta(s)$ denotes Riemann's zeta function [3, $\S 1.12]$. These results are inserted into (2.3). Then, after some rearrangement and simplification, we find

$$
\begin{aligned}
\chi_{n}(z)= & \sum_{k=0}^{n-2}\left(1-2^{-n+k}\right) \zeta(n-k) \frac{(\log z)^{k}}{k !} \\
& +\sum_{k=0}^{\infty} \frac{(-1)^{k}}{(2 k+2)_{n}}\left(1-2^{-2 k-1}\right) \zeta(2 k+2) \frac{(\log z)^{n+2 k+1}}{\pi^{2 k+2}} \\
& +\frac{(\log z)^{n-1}}{2(n-1) !}\left[\psi(n)-\psi\left(\frac{1}{2}\right)-\log (-2 \log z)\right], \quad|\log z|<\pi,
\end{aligned}
$$

where $(2 k+2)_{n}$ is a short notation for

$$
(2 k+2)_{n}=(2 k+2)(2 k+3) \cdots(2 k+n+1) .
$$

The infinite series in (2.4) obviously converges if $|\log z| \leq \pi$.

For $z=e^{i \alpha}, \alpha \in \mathbb{R}$, the expansion (2.4) becomes

$$
\begin{aligned}
& \chi_{n}\left(e^{i \alpha}\right)= \sum_{k=0}^{n-2}\left(1-2^{-n+k}\right) \zeta(n-k) \frac{(i \alpha)^{k}}{k !} \\
&+i^{n+1} \sum_{k=0}^{\infty} \frac{1-2^{-2 k-1}}{(2 k+2)_{n}} \zeta(2 k+2) \frac{\alpha^{n+2 k+1}}{\pi^{2 k+2}} \\
&+\frac{(i \alpha)^{n-1}}{2(n-1) !}\left[\psi(n)-\psi\left(\frac{1}{2}\right)-\log (2|\alpha|)+\frac{1}{2} \pi i \operatorname{sgn}(\alpha)\right], \\
&|\alpha| \leq \pi
\end{aligned}
$$

By taking real and imaginary parts in (2.5), similar expansions are obtained for the functions $C_{n}(\alpha)$ and $S_{n}(\alpha)$, introduced in (1.6). Notice that the real (imaginary) part of (2.5) is a polynomial in $\alpha$, if $n$ is even (odd). Thus the Fourier series (1.6) for $C_{2 n}(\alpha)$ and $S_{2 n+1}(\alpha)$ are summable in closed form, the sum being a polynomial in $\alpha$ on the intervals $[0, \pi]$ and $[-\pi, 0]$. These results also follow from the known Fourier expansions of the Bernoulli polynomials (see [3, equations 1.13(14), (15)]). 
The expansions (2.4) and (2.5) are perfectly suitable for the numerical evaluation of $\chi_{n}(z)$ in the two cases of real $z \lesssim 1$, and $z=e^{i \alpha}, \alpha \in \mathbb{R}$. Here, the necessary values of $\zeta(n-k)$ and $\zeta(2 k+2)$ can be taken from [1, Table 23.3]. From [3, equation 1.13(22)] we also have

$$
\zeta(2 k+2)=\frac{(-1)^{k}(2 \pi)^{2 k+2}}{2(2 k+2) !} B_{2 k+2}
$$

where the Bernoulli numbers $B_{2 k+2}$ are rational numbers, listed in [1, Table 23.2]. On substitution of (2.6) into (2.4) and (2.5), it is recognized that the coefficients of the series become rational numbers. The expansion (2.4) is convergent for real $z$ with $e^{-\pi}=0.0432 \leq z \leq 1$. To achieve rapid convergence, we suggest to restrict the range to $e^{-\pi / 2}=0.208 \leq z \leq 1$. Then in the worst case $z=e^{-\pi / 2}$, the general term of the series in (2.4) behaves like

$$
\frac{1-2^{-2 k-1}}{(2 k+2)_{n}} \zeta(2 k+2) \frac{(\pi / 2)^{n+2 k+1}}{\pi^{2 k+2}} \sim \frac{(\pi / 2)^{n-1}}{(2 k+2)_{n}} \frac{1}{2^{2 k+2}} \quad(k \rightarrow \infty) .
$$

Thus, the tail of the series converges like a geometric series of ratio $\frac{1}{4}$, and the truncation error is therefore less than $\frac{4}{3}$ times the first term discarded. Over the remaining range, $0 \leq z \leq 0.208$, the function $\chi_{n}(z)$ can be calculated from the original series (1.3). Alternatively, one might place the transition point at $z_{0}=0.34$ instead of 0.208 . This value has been chosen such that for $z=z_{0}$ both series (1.3) and (2.4) converge like a geometric series of ratio 0.12. As for the functions $C_{n}(\alpha)$ and $S_{n}(\alpha)$, we establish the symmetry properties: $C_{n}(\alpha)$ is even in $\alpha ; S_{n}(\alpha)$ is odd in $\alpha ; C_{n}(\alpha)=-C_{n}(\pi-\alpha) ; S_{n}(\alpha)=S_{n}(\pi-\alpha)$. Because of these properties it is sufficient to calculate $C_{n}(\alpha)$ and $S_{n}(\alpha)$ over the range $0 \leq \alpha \leq \pi / 2$ only. The calculation is readily made by use of the real and imaginary parts of the expansion (2.5). In the worst case $\alpha=\pi / 2$, one has again the estimate (2.7) for the general term of the series in (2.5).

\section{SPEcial CASES $n=2$ AND $n=3$}

The series (1.1) and (1.2) are identical to $\chi_{2}(A), \chi_{3}(A)$ and $S_{2}(\alpha), C_{3}(\alpha)$, respectively. The numerical evaluation of these slowly convergent series was recently discussed by Dempsey, Liu, and Dempsey [2]. These authors used Plana's summation formula (PSF) along with Romberg integration to improve the convergence of the series under consideration. In this section it is shown that the expansions (2.4) and (2.5), specialized for the cases $n=2$ and $n=3$, provide a better alternative for the numerical calculation.

In the special cases $n=2$ and $n=3$, the expansion (2.4), with $z$ replaced by $A$, reduces to

$$
\begin{aligned}
\chi_{2}(A)= & \frac{\pi^{2}}{8}+\sum_{k=0}^{\infty} \frac{(-1)^{k}}{(2 k+2)(2 k+3)}\left(1-2^{-2 k-1}\right) \zeta(2 k+2) \frac{(\log A)^{2 k+3}}{\pi^{2 k+2}} \\
& +\frac{1}{2} \log A[1+\log 2-\log (-\log A)], \quad e^{-\pi} \leq A \leq 1,
\end{aligned}
$$




$$
\chi_{3}(A)=\frac{7}{8} \zeta(3)+\frac{\pi^{2}}{8} \log A
$$

$$
\begin{aligned}
& +\sum_{k=0}^{\infty} \frac{(-1)^{k}}{(2 k+2)(2 k+3)(2 k+4)}\left(1-2^{-2 k-1}\right) \zeta(2 k+2) \frac{(\log A)^{2 k+4}}{\pi^{2 k+2}} \\
& +\frac{1}{4}(\log A)^{2}\left[\frac{3}{2}+\log 2-\log (-\log A)\right], \quad e^{-\pi} \leq A \leq 1 .
\end{aligned}
$$

The expansion (3.1) can also be derived from (1.4) and the expansion of the dilogarithm $\mathrm{Li}_{2}\left(e^{-z}\right)$ in [5, p. 21]. The expansions (3.1) and (3.2) are very suitable for the numerical evaluation of $\chi_{2}(A)$ and $\chi_{3}(A)$ over the range $e^{-\pi / 2} \leq$ $A \leq 1$, say. By taking into account ten terms $\left(\sum_{k=0}^{9}\right)$ of the series in (3.1) and (3.2), the truncation error will be less than $7.4 \times 10^{-10}$ if $n=2$, and less than $4.8 \times 10^{-11}$ if $n=3$, in the worst case $A=e^{-\pi / 2}$. Numerical comparisons were made with the PSF procedure in [2], for $A=\pi / 4$. In [2, Tables 8 and 10], $\chi_{2}(\pi / 4)$ and $\chi_{3}(\pi / 4)$ were calculated by PSF and Romberg integration, requiring 512 subdivisions of the integration intervals for 15 decimal place accuracy. On the other hand, for $A=\pi / 4$ the expansions (3.1) and (3.2) have truncation errors less than $6.6 \times 10^{-17}$ and $3.2 \times 10^{-16}$, when the series are truncated to five and four terms, respectively. Clearly, the computational procedure based on the expansions (3.1) and (3.2) is undeniably superior. The values of $\zeta(2 k+2)$ needed in (3.1) and (3.2) can be found from (2.6) and [1, Table 23.2]; as before, the coefficients of the series in (3.1) and (3.2) become rational numbers.

Next we turn to the numerical evaluation of the series (1.2), which are identical to $S_{2}(\alpha)$ and $C_{3}(\alpha)$. By taking the real and imaginary parts of the expansion (2.5) with $n=2,3$, we find

$$
\begin{gathered}
C_{2}(\alpha)=\frac{\pi^{2}}{8}-\frac{\pi}{4}|\alpha|, \quad|\alpha| \leq \pi, \\
S_{2}(\alpha)=-\sum_{k=0}^{\infty} \frac{1-2^{-2 k-1}}{(2 k+2)(2 k+3)} \zeta(2 k+2) \frac{\alpha^{2 k+3}}{\pi^{2 k+2}} \\
+\frac{1}{2} \alpha[1+\log 2-\log |\alpha|], \quad|\alpha| \leq \pi, \\
C_{3}(\alpha)=\frac{7}{8} \zeta(3)+\sum_{k=0}^{\infty} \frac{1-2^{-2 k-1}}{(2 k+2)(2 k+3)(2 k+4)} \zeta(2 k+2) \frac{\alpha^{2 k+4}}{\pi^{2 k+2}} \\
-\frac{1}{4} \alpha^{2}\left[\frac{3}{2}+\log 2-\log |\alpha|\right], \quad|\alpha| \leq \pi, \\
S_{3}(\alpha)=\frac{\pi^{2}}{8} \alpha-\frac{\pi}{8} \alpha|\alpha|, \quad|\alpha| \leq \pi .
\end{gathered}
$$

The closed-form results for $C_{2}(\alpha)$ and $S_{3}(\alpha)$ are known (see [2, equation $\left.\left.\left(1 a^{\prime}, b^{\prime}\right)\right]\right)$. The expansion (3.4) can also be derived from (1.7) and the expansion of Clausen's function $\mathrm{Cl}_{2}(\theta)$ in [5, p. 106]. The expansions (3.4) and (3.5) are again eminently suitable for the numerical evaluation of $S_{2}(\alpha)$ and 
$C_{3}(\alpha)$ over the range $0 \leq \alpha \leq \pi / 2$. By taking into account ten terms of the series in (3.4) and (3.5), the truncation error is of the order of $10^{-9}$ to $10^{-10}$, in the worst case $\alpha=\pi / 2$. Numerical comparisons were made with the PSF procedure in [2], for $\alpha=\pi / 4$ (see [2, Table 3]) and $\alpha=j \pi / 18, j=1,2, \ldots, 9$ (see [2, Table 11]). As indicated in [2, Tables 3 and 11], the calculation by PSF and Romberg integration required 64 and 128 subdivisions of the integration intervals, respectively, for 15 decimal place accuracy. For $\alpha=\pi / 4$ the expansion (3.4) has a truncation error less than $9.4 \times 10^{-17}$, when the series is truncated to ten terms. For $\alpha=j \pi / 18$ the expansion (3.5) has 14 decimal place accuracy or better, if truncated to a number of terms that increases monotonically from 3 for $j=1$ to 16 for $j=9$.

Finally, it is interesting to mention that the analysis in [2] got very close to the expansion (3.5). In [2, equations (17), (18)] there is an asymptotic expansion due to Grossman, which reads

$$
C_{3}(t)=\sum_{k=0}^{\infty} \frac{\cos (2 k+1) t}{(2 k+1)^{3}} \sim \frac{7}{8} \zeta(3)-\phi(t)+\frac{1}{8} \phi(2 t) \quad(t \rightarrow 0+),
$$

where

$$
\phi(t)=\frac{t^{2}}{2}\left[\log \left(\frac{1}{t}\right)+\frac{3}{2}\right]+\sum_{j=2}^{\infty} \frac{B_{2 j-2}}{(2 j-2)(2 j) !} t^{2 j} .
$$

To enable a comparison with (3.5), we set, by use of (2.6),

$$
B_{2 j-2}=(-1)^{j}(2 \pi)^{-2 j+2} 2(2 j-2) ! \zeta(2 j-2) ;
$$

then it follows that

$$
\begin{aligned}
-\phi(t)+\frac{1}{8} \phi(2 t)= & -\frac{1}{4} t^{2}\left[\frac{3}{2}+\log 2-\log t\right] \\
& +\sum_{j=2}^{\infty} \frac{(-1)^{j}\left(1-2^{-2 j+3}\right)}{(2 j-2)(2 j-1)(2 j)} \zeta(2 j-2) \frac{t^{2 j}}{\pi^{2 j-2}} .
\end{aligned}
$$

The latter series agrees with the series in $(3.5)$ except for the factor $(-1)^{j}$, which is erroneous. The error can easily be corrected by inserting a factor $(-1)^{j}$ in the series in (3.8). This also explains the conclusion in [2, p. 700]: Grossman's "expression in (17) is exact for $t=0$, accurate to eight decimal places for $t=\pi / 18$, but accurate to only three decimal places for $t=4 \pi / 9$ (see Table 11)." The corrected expression is exact for all $t$ in the range $0 \leq t \leq \pi$, and is not only an asymptotic expansion but a convergent series expansion.

\section{BIBLIOGRAPHY}

1. M. Abramowitz and I. A. Stegun, Handbook of mathematical functions, Dover, New York, 1965.

2. K. M. Dempsey, D. Liu, and J. P. Dempsey, Plana's summation formula for $\sum_{m=1,3}^{\infty}, m^{-2} \sin (m \alpha), m^{-3} \cos (m \alpha), m^{-2} A^{m}, m^{-3} A^{m}$, Math. Comp. 55 (1990), 693-703.

3. A. Erdélyi, W. Magnus, F. Oberhettinger, and F. G. Tricomi, Higher transcendental functions, Vol. I, McGraw-Hill, New York, 1953.

4. W. Gautschi, On certain slowly convergent series occurring in plate contact problems, Math. Comp. 57 (1991), 325-338. 
5. L. Lewin, Polylogarithms and associated functions, North-Holland, New York, 1981.

6. $\mathrm{H}$. Li, Unbonded contact of rectangular plates on edge supports or elastic foundations, Ph.D. Thesis, Clarkson University, Potsdam, 1988.

Department of Mathematics and Computing Science, Eindhoven University of TechNOLOGY, P. O. Box 513, 5600 MB EINDHOVEN, THE NETHERLANDS

E-mail address: wstanal@win.tue.nl

Department of Civil and Environmental Engineering, Clarkson University, Potsdam, NEW YORK 13699-5710

E-mail address: jdempsey@jpd3m.cee.clarkson.edu 